\title{
Patrón clínico y citogenético en pacientes con síndrome mielodisplásico en Cúcuta (Norte de Santander, Colombia)
}

\section{Clinical and cytogenetic pattern in patients with myelodysplasic syndrome in Cúcuta (Norte de Santander, Colombia)}

\footnotetext{
- Juan Carlos Serrano1, Carlos Roberto Varón Jaimes², Ana Teresa Govin³ , Gabriel Celis , Luz Karine Maldonado5, Javier Pacheco ${ }^{6}$

' Médico cirujano, especialista en Medicina Interna y Hematología. Unidad Hematológica Especializada. Cúcuta (UHE) (Colombia).

${ }_{2}^{2}$ Médico cirujano, especialista en Medicina Interna y Hematología. Director Médico, Unidad Hematológica Especializada. Cúcuta (UHE) (Colombia).

${ }^{3}$ Médico cirujano, especialista en Medicina Interna y Hematología. Servicio de Hematología, Unidad Hematológica Especializada. Cúcuta (UHE) (Colombia).

${ }^{4}$ Bacteriólogo. Departamento de Citogenética, Unidad Hematológica Especializada. Cúcuta (UHE) (Colombia).

${ }^{5}$ Bacterióloga. Departamento de Citogenética, Unidad Hematológica Especializada. Cúcuta (UHE) (Colombia).

${ }^{6}$ Médico cirujano, especialista en Medicina Interna y Oncohematología. Departamento de Oncología, Hospital San José de Bogotá.
}

\section{Resumen}

Los síndromes mielodisplásicos (SMD) son neoplasias clonales de pronóstico variable de acuerdo con estratificación. La citogenética es clave en diagnóstico y pronóstico.

Población y métodos: en UHE estudiamos 81 pacientes con SMD. Caracterización clínica, laboratorio, IPSS-R, mortalidad, análisis citogenético con descripción de anomalías clonales típicas y atípicas de SMD.

Resultados: la media etaria fue de 71 años, con predominio femenino. El subtipo más frecuente fue SMD con displasia multilinaje y displasia unilineal, y la manifestación más común fue anemia. Hubo mayor proporción de casos con riesgo bajo e intermedio (IPSS-R). La media de seguimiento no mostró diferencias entre grupos $(p=0,18)$. La sobrevida global a cinco años fue del 79\%. La progresión a LMA se dio en un $9,8 \%$. La mayor cantidad de decesos fueron en grupos de muy alto (85\%) y alto riesgo (50\%). La citogenética se presentó con el $72 \%$ de índice mitótico en 24 horas. El cariotipo clonal fue del $43 \%$ con 53 alteraciones de 81 estudios, siendo complejo en un 8,6\%. Las anomalías numéricas y estructurales más frecuentes fueron: del(5q) y -Y 6,1\%, del(20q), del(11q), del(17p) y +8 en el 3,7\%. También, encontramos anomalías citogenéticas no típicas de SMD, como -22, -13-11, del(10q), 14p+, 15q+, en el 1,2\%.

Conclusiones: subtipos de SMD, citopenias, IPSS-R, mortalidad, alteraciones citogenéticas estructurales y numéricas guardan correlación con la literatura. La presencia de alteraciones citogenéticas atípicas plantea mayor evaluación y expansión en bases de datos internacionales.

Palabras clave: síndrome mielodisplásico, citogenética, Colombia.

\begin{abstract}
Myelodysplastic syndromes (MDS) are clonal neoplasms of variable prognosis according to stratification. Cytogenetics is key in diagnosis and prognosis.

Population and methods: At UHE we studied 81 patients with MDS. Clinical characterization, laboratory, IPSS-R, mortality, cytogenetic analysis with description of typical clonal anomalies and atypical SMD.

Results: Mean age 71 years, with female predominance. Most frequent subtype was SMD with multilineage dysplasia and unilineal dysplasia, anemia was the most common manifestation. Higher proportion
\end{abstract}


of low and intermediate risk cases (IPSS-R). Mean follow-up did not show differences between groups ( $p=0.18$ ). Overall 5 -year survival was $79 \%$. Progression to AML in $9.8 \%$. Increased number of deaths in very high (85\%) and high risk (50\%) groups. Cytogenetics with $72 \%$ mitotic index at 24 hours. Clonal karyotype $43 \%$ with 53 alterations of 81 studies, being complex in $8.6 \%$. (5q) and $-Y 6.1 \%$, (20q), (11q), (17p) and +8 in 3.7\%. We found cytogenetic abnormalities not typical of MDS as: $-22,-13-11$, from (10q), $14 p+, 15 q+$ at $1.2 \%$.

Conclusions: Subtypes of MDS, cytopenias, IPSS-R, mortality, structural and numerical cytogenetic alterations; correlate with the literature. Presence of atypical cytogenetic alterations deserves more evaluation and expansion in international database.

Key words: Myelodysplastic syndrome, cytogenetics, Colombia.

\section{Introducción}

Los síndromes mielodisplásicos (SMD) son un grupo heterogéneo de trastornos neoplásicos clonales hematopoyéticos caracterizados por hemopoyesis ineficaz y displasia, con reducción cuantitativa y funcional de glóbulos rojos, leucocitos y plaquetas, resultando en manifestaciones clínicas de anemia, sangrados e infecciones ${ }^{1,2}$. Los SMD ocurren más comúnmente en adultos mayores y pueden ocurrir de novo o surgir después de exposición a terapia mutagénica (radiación, quimioterapia, inmunosupresores), resultando en SMD secundario con selección de clones hematopoyéticos resistentes a terapia ${ }^{3,4}$.

El diagnóstico debe considerarse en caso de citopenia(s) inexplicada(s). Es importante la integración diagnóstica con evaluación del frotis de sangre periférica y aspirado medular para documentar líneas displásicas hematopoyéticas; además, evaluar alteraciones clonales citogenéticas, en citometría de flujo y nivel de biología molecular 5 . La patobiología de los SMD es compleja y no completamente entendida, se han implicado alteraciones en la función del microambiente medular o en sus células troncales ${ }^{6}$. El desarrollo de SMD implica una serie de cambios genéticos que comprometen el crecimiento y la diferenciación de células hematopoyéticas, dando como resultado una acumulación de células mieloides anormales e inmaduras y la consiguiente falla medular, y en una segunda fase acumular daño clonal, que podría desencadenar leucemia aguda7,8. Los avances en la identificación de anomalías cromosómicas y genéticas recurrentes han proporcionado información sobre la patobiología de los SMD, capaz de identificar pacientes con mayor probabilidad de desarrollar LMA, ya sea en casos de SMD primario o secundario a quimioterapia o radioterapia ${ }^{9-11}$.
Se debe destacar que cerca del $25 \%$ al $70 \%$ de las pruebas citogenéticas procesadas en otras localidades de Colombia son informadas como normales, afectando el valor diagnóstico y pronóstico por este medio ${ }^{12}$. En UHE se realizan estudios en el laboratorio de genética de nuestra institución, aportando al escenario local herramientas clave en el estudio de la enfermedad, considerando la optimización del recurso con base en la calidad de la muestra, transporte y tiempo de procesamiento.

Son nuestros objetivos en el presente estudio describir las variables clínicas, identificar subtipos más frecuentes de SMD, estratificación por IPSS-R ${ }^{13}$, y evaluar mortalidad por grupos. En el área paraclínica, establecer calidad en muestras citogenéticas con índice mitótico a las 24 y 72 horas, reporte de cariotipos normales y proporción de aquellos con expresión clonal, frecuencias de anomalías cromosómicas numérica y estructural propias de SMD, así como documentación de anomalías citogenéticas poco frecuentes, haciendo comparación con datos nacionales e internacionales.

\section{Población y métodos}

Fueron evaluadas historias clínicas electrónicas de manual de procesos operativos (MPO, 03-F-03-3) de pacientes procedentes de la Unidad Hematológica Especializada en La Riviera, Cúcuta (Norte de Santander), de manera retrospectiva, durante un período de ocho años, desde el año 2009 hasta 2017, en forma de un estudio descriptivo de una serie de casos, ingresados en modo consecutivo, en muestreo por conveniencia, seleccionando casos que cumplieran criterios de inclusión y exclusión. 


\section{Criterios de inclusión}

- $\quad$ Edad mayor de 18 años con citopenias inexplicables en uno o más contajes sanguíneos y elementos de la médula ósea, con valores definidos en: hemoglobina $<10 \mathrm{~g} / \mathrm{dl}(100 \mathrm{~g} / \mathrm{l})$; recuento absoluto de neutrófilos $<1,8 \times 109 /$ l (<1.800/microl); plaquetas $<100 \times 109 / /$ $(<100.000 /$ microl). Displasia significativa $(\geq 10 \%$ de precursores eritroides, granulocitos o megacariocitos) mediante estudio de extendido de frotis de sangre periférica, aspiración de médula ósea y biopsia de médula ósea en ausencia de otras causas de displasia. Citometría de flujo: fenotipo aberrante en $\mathrm{MO}$ sugestivo de clonalidad (criterios de Ogata y OMS 2016). Estudio citogenético de UHE procesados en período menor a seis horas, bajo metodología estandarizada del American College of Medical Genetics y del ISCN ${ }^{14-16}$. Estudios FISH $(-7,5 q-, 20 q-)$.

\section{Criterios de exclusión}

Casos pediátricos, LMA por contaje de blastos mayor al $19 \%$ en SP o MO, presencia de sarcoma granulocítico o ciertas anomalías genéticas: $\mathrm{t}(8 ; 21)$, inv(16) y t(15;17) son diagnóstico de LMA, independiente del recuento de blastos ${ }^{10}$; pacientes con criterios de ICUS 'citopenia idiopática de significado indeterminado' o de CHIP 'hematopoyesis clonal de potencial indeterminado'; uso de fármacos como el factor estimulante de colonias de granulocitos, ácido valproico, micofenolato, ganciclovir y alemtuzumab, etc.; presencia de $\mathrm{VIH}$, exceso de zinc, déficit de cobre, anemias megaloblásticas, LMC, neoplasias mielodisplásicas/mieloproliferativas.

Los pacientes confirmados como SMD fueron clasificados bajo el sistema de la Organización Mundial de la Salud (actualización 20165) (ver tabla 1). Así mismo, se estratificó por Sistema de Puntuación Pronóstica Internacional (Revised International Prognostic Scoring System ${ }^{13}$. Se establecieron los antecedentes de exposición a tóxicos (alcohol, tabaco, bencenos, etc.) y si había tratamiento quimioterápico previo en contexto de patología hematooncológica previa. Se investigó uso de agentes terapéuticos EPO, GM-CSF, azacitidina, decitabina y lenalidomida, especificando el número de ciclos y respuesta. Se determinó sobrevida global a cinco años en grupos de bajo y alto riesgo de IPSS-R. Se analizan variables en frecuencias y comparación de medias por Anova. Los datos fueron estudiados con paquete estadístico SPSS 19.

\section{Resultados}

De 106 pacientes evaluados con citopenias inexplicables para ser incluidos en el protocolo, se descartaron 17 pacientes por no cumplir con los criterios de inclusión (ver figura 1).

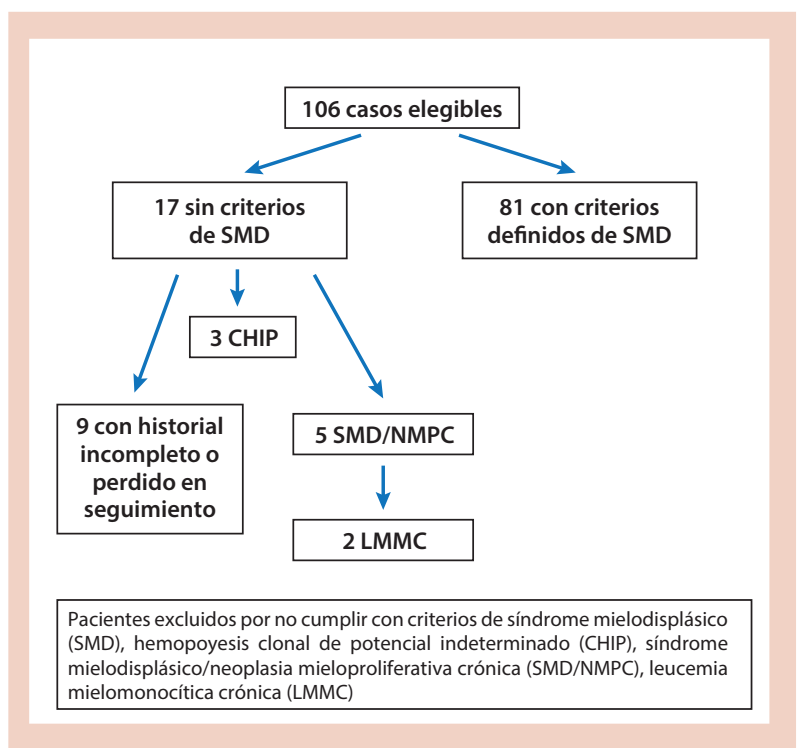

Figura 1. Número de casos con citopenias sospechosas de síndrome mielodisplásico evaluados en Unidad Hematológica Especializada.

Se encontró un total de 81 pacientes con criterios de síndrome mielodisplásico, siendo 47 pacientes femeninos (58\%) y 34 masculinos (42\%), con media de edad $71 \pm 12,60$ años (rango de 37-85, primer cuartil en 60, tercer cuartil en 78), donde el 77,7\% tenía edad mayor a 60 años. La manifestación clínica predominante fue la anemia en el $91 \%$ (74 casos), pancitopenia en el 18,5\% (15 casos), trombocitopenia aislada en el 4,9\% (4 casos) y leucopenia aislada en el 7,4\% (6 casos). Los subtipos de la Organización Mundial de la Salud más frecuentes fueron: SMD con displasia multilinaje, SMD con displasia unilinaje anemia refractaria y SMD no clasificable. La distribución de casos se presenta en la tabla 1.

Con respecto a factores de riesgo por exposición a agentes tóxicos, es importante destacar que un 16\% (13 casos) fueron síndromes mielodisplásicos secundarios a quimioterapia, radioterapia e inmunosupresores; y un $7 \%$ (6 casos) se relacionó con la exposición a bencenos.

\section{Alteraciones citogenéticas}

Es esencial destacar que en 77 de 81 de los casos donde se reporta el número de metafases procesadas, se pudieron analizar 20 metafases a las 24 horas en 
Tabla 1. Distribución de casos de SMD en UHE según clasificación OMS (81 casos) 2016

\begin{tabular}{|l|c|c|}
\hline \multicolumn{1}{|c|}{ Subtipos SMD según OMS 2016 en UHE } & Casos & $\%$ \\
\hline SMD con displasia de una sola serie & 22 & 27,1 \\
\hline Anemia refractaria & 18 & 22,2 \\
\hline Leucopenia refractaria & 2 & 2,4 \\
\hline Trombocitopenia refractaria & 2 & 2,4 \\
\hline SMD con displasia multilinaje & 40 & 49,3 \\
\hline SMD con anillos sideroblásticos y displasia unilinaje (anemia) & 1 & 1,2 \\
\hline SMD con anillos sideroblásticos y displasia multilinaje & 1 & 1,2 \\
\hline SMD con exceso de blastos I & 3 & 3,7 \\
\hline SMD con exceso de blastos II & 6 & 7,4 \\
\hline SMD inclasificable & 5 & 6,1 \\
\hline SMD con delección aislada de 5q- & 3 & 3,7 \\
\hline Total & $\mathbf{8 1}$ & $\mathbf{1 0 0}$ \\
\hline
\end{tabular}

cultivo sin estímulo, en el 72,7\% de los casos, mostrando un alto índice mitótico. El hallazgo más frecuente fue el cariotipo normal en el 56,7\%, encontrándose un $43 \%$ (35) de cariotipos anormales con criterios de clonalidad, con un total de 53 alteraciones en 81 muestras procesadas. El cariotipo complejo se pudo documentar en el 8,6\% (7 casos). Se pudo así mismo documentar otras anomalías citogenéticas numéricas y estructurales típicas de SMD, siendo más común el del(5q) y -Y en el 6,1\%, seguidos por del(20q), del(11q), del(17p) y trisomía del 8 en el 3,7\%. Los datos se resumen en la tabla 2.

Se pudieron hallar también otras anomalías citogenéticas no típicas de SMD, como -22, -13-11, del(10q), $14 p+, 15 q+$ en el $1,2 \%$, siendo de baja frecuencia. Se evidenció en un caso una t(8:21) y dos con inv(16), que se consideraron SMD con evolución clonal a leucemia, que finalmente se transformaron en LMA. Desde el

Tabla 2. Anomalías típicas de síndrome mielodisplásico encontradas en 31 pacientes con cariotipo clonal sobre el total de los 81 casos evaluados en UHE

\begin{tabular}{|l|c|c|}
\hline \multicolumn{1}{|c|}{ Anomalías CG } & $\%$ & N casos \\
\hline$-Y$ & 6,1 & 5 \\
\hline $\operatorname{del}(5 q)$ & 6,1 & 5 \\
\hline $\operatorname{del}(20 q)$ & 3,7 & 3 \\
\hline $\operatorname{del}(11 q)$ & 3,7 & 3 \\
\hline $\operatorname{del}(17 q)$ & 3,7 & 3 \\
\hline+8 & 3,7 & 3 \\
\hline $\operatorname{del}(12 p)$ & 2,4 & 2 \\
\hline+19 & 1,2 & 1 \\
\hline $\operatorname{del}(9 q)$ & 1,2 & 1 \\
\hline $\operatorname{del}(7 q)$ & 1,2 & 1 \\
\hline$t(1 ; 3)$ & 1,2 & 1 \\
\hline $\ln (3) / d e l(3 q)$ & 1,2 & 2 \\
\hline-17 & 2,4 & \\
\hline
\end{tabular}

punto de vista molecular, se pudo confirmar en tres casos del(5q) aislado, en otro se asoció a cariotipo complejo y en otro el FISH no fue realizado.

\section{Estratificación pronóstica en SMD}

Bajo sistema de puntuación pronóstica internacional IPSS-R, se encontró mayor número de casos en riesgo bajo, con un 33,3\% (27), y riesgo intermedio, con el 24,6\% (20); al realizar análisis de medias de seguimiento en meses por Anova de una vía, no se apreciaron diferencias estadísticamente significativas entre grupos ( $p$ $=0,18)$. Los datos en detalle se expresan en la tabla 3 .

Tabla 3. Estratificación de riesgo IPSS-R, seguimiento en meses y mortalidad por grupos de riesgo en 81 casos de SMD en UHE

\begin{tabular}{|l|c|c|c|c|c|}
\hline \multicolumn{1}{|c|}{ Estrato IPSS-R } & $\begin{array}{c}\text { Muy } \\
\text { bajo } \\
\text { riesgo }\end{array}$ & $\begin{array}{c}\text { Bajo } \\
\text { riesgo }\end{array}$ & $\begin{array}{c}\text { Riesgo } \\
\text { inter- } \\
\text { medio }\end{array}$ & $\begin{array}{c}\text { Alto } \\
\text { ries- } \\
\text { go }\end{array}$ & $\begin{array}{c}\text { Muy alto } \\
\text { riesgo }\end{array}$ \\
\hline N casos & 17 & 27 & 20 & 10 & 7 \\
\hline Proporción por grupo \% & $20,8 \%$ & $33,3 \%$ & $24,6 \%$ & $12,3 \%$ & $8,6 \%$ \\
\hline $\begin{array}{l}\text { Media de seguimiento } \\
\text { (meses) }\end{array}$ & $\begin{array}{c}30,5 \pm \\
30,2\end{array}$ & $\begin{array}{c}25,2 \pm \\
31,9\end{array}$ & $\begin{array}{c}21 \pm \\
21\end{array}$ & $\begin{array}{c}18,5 \pm \\
15,3\end{array}$ & $\begin{array}{c}11,8 \pm \\
6,9\end{array}$ \\
\hline Mortalidad por grupo \% & $14 \%$ & 0 & $20 \%$ & $50 \%$ & $85 \%$ \\
\hline
\end{tabular}

\section{Tratamiento SMD en UHE}

La modalidad terapéutica más utilizada fue la eritropoyetina en el $54,3 \%$ (44 casos) con tasas de respuestas del 75\%. La terapia hipometilante más empleada fue la 5 -azacitidina en el 27,1\% (22 casos), con una mediana de ciclos empleados de $8 \pm 2,32$, en contraste con la decitabina en tan solo 3 casos (3,7\%), mediana de ciclos $6 \pm 2,2$. La lenalidomida se usó en 3 casos (3,7\%), mediana de $12 \pm 2$ y romiplostim en 3 casos. En la tabla 4 se resumen las modalidades terapéuticas empleadas.

En el seguimiento a 30 meses de los 81 pacientes estudiados, 17 (20,9\%) habían fallecido: el 9,8\% (8 casos) presentó falla medular, el 6,1\% (5 casos) tuvo evolución a leucemia mieloide aguda y el 4,9\% (4 casos) fue por otras causas. La mayor mortalidad se apreció en el grupo de alto muy alto riesgo (85\%), alto riesgo (50\%), intermedio (20\%), muy bajo riesgo (14\%) (muertes no asociadas a SMD) y bajo riesgo (0\%). La sobrevida global del grupo

Tabla 4. Opciones terapéuticas utilizadas en 75 casos de SMD en UHE con indicación de tratamiento, de un total de 81 casos (6 pacientes sin criterios para tratamiento)

\begin{tabular}{|l|c|c|}
\multicolumn{1}{|c|}{ Tratamiento } & $\%$ & Casos \\
\hline Eritropoyetina & $54,3 \%$ & 44 \\
\hline 5-azacitidina & $27,1 \%$ & 22 \\
\hline Decitabina & $3,7 \%$ & 3 \\
\hline Lenalidomida & $3,7 \%$ & 3 \\
\hline Romiplostim & $3,7 \%$ & 3 \\
\hline
\end{tabular}


a 30 meses fue del 79\%. En los pacientes fallecidos, el $35 \%$ (6) tenía cariotipo normal; el 23\% (4), cariotipo complejo; y el $11 \%(2)$, del20 e -y.

\section{Discusión}

Existen muy pocos datos epidemiológicos y estadísticos en relación con el síndrome mielodisplásico en Colombia, no obstante la media de edad de nuestra serie fue de 71 años, semejante a la media de 75 años reportada por Mora y colaboradores en un grupo de 39 pacientes con SMD del Hospital San José de Bogotá entre 2010 y 2014'2, también similar a lo revelado por un grupo argentino con 70 años $^{17}$, pero estas medias son discretamente superiores a las descritas en Norteaméri$\mathrm{ca}$, que se ubican en 65 años $^{18}$. Esto se pudiese explicar por una demora al acceso de consultas especializadas en países latinoamericanos, en especial en Colombia, con mayores procesos administrativos. En cuanto al sexo, en la mayoría de reportes se reseña un predominio del sexo masculino entre el $55 \%$ al $62 \%^{12,17-19}$. En contraste con nuestra serie, con predominio del sexo femenino (58\%), fenómeno explicable por cambios de la demografía regional del Norte de Santander, con una predominancia del sexo femenino, debido a incremento de muertes por violencia en el contexto de conflictos armados en Colombia. En Latinoamérica solo el grupo chileno ha mostrado un predominio del sexo femenino ${ }^{20}$.

Con respecto al subtipo de SMD según la clasificación de la OMS, no se mostró diferencia en relación con estadísticas globales en lo referente a SMD con displasia multilinaje $(51,8 \%)$ y SMD con displasia unilinaje $(28,3 \%)$ y anemia refractaria $(22,2 \%)^{2-6}$. Sin embargo, se observó baja incidencia de SMD EB 1 y 2 (3,7\% y $7,4 \%)$, con una alta frecuencia de SMD no clasificado con variante hipocelular (7,4\%), que contrasta con recientes publicaciones de frecuencias en subtipos OMS del grupo de Dusseldorf y suramericano ${ }^{19,20}$, con SMD EB 1y 2 en re (16-17\%), lo cual se podría correlacionar en Colombia con los retardos en el diagnóstico y procesal en nuestro medio, que llevaría a encontrar mayor número de leucemias mieloides en adultos mayores por transformación de SMD de alto riesgo. En nuestra serie se apreció el 16\% de casos de SMD secundario a tratamiento citotóxico o radioterapia, que es comparable a otras series ${ }^{18-20}$. De este mismo modo, la distribución de casos de acuerdo con la estratificación de IPSS-R mostró una mayor proporción entre casos de riesgo bajo e intermedio (33\%-29\%), seguido de riesgo muy bajo y alto riesgo, siendo muy similar a la estadística mundia|15-19.

Desde el punto de vista terapéutico, se apreció un empleo de eritropoyetina en un 54,3\% (44 casos), con una media de dosis de 10 mil unidades semanales, observando altas tasas de respuestas a dosis intermedias entre 8 mil y 12 mil unidades semanales. La tasa de empleo en nuestro grupo es inferior a lo reportado por las estadísticas de usuarios en Medicare en los Estados Unidos en la primera década de 2000, que mostraba una frecuencia de utilización en el $62 \%$ de los casos, aunque era discutible la adherencia a las guías en esa serie $^{21}$. El segundo tratamiento más usado fue la terapia hipometilante en el 27\% (22 casos), con media de ciclos de 8 y tasas de respuesta globales del $22 \%$, que está en relación con experiencias en el mundo real con azacitidina, como se apreció en el estudio alemán con seguimiento de 77 casos de 2004 a 2015, con media de ciclos de 6 y tasa de respuesta del $18 \%^{22}$.

Las alteraciones citogenéticas en nuestro estudio evidenciaron cariotipo normal en el 56,7\% (46 casos), contra cariotipo patológico en el $43 \%$ (35 casos), lo que fue muy superior a la proporción de casos descrita por otro estudio realizado en Bogotá, que reportó el 26\% ${ }^{12}$. En este aspecto es importante destacar la calidad del reporte citogenético, el factor transporte y tiempo de procesamiento, que pudiese incidir negativamente en los resultados de los estudios; recordemos que las anomalías cromosómicas suelen determinarse entre el 50\% al $60 \%$ de los pacientes con síndrome mielodisplásico a nivel mundial, de allí su papel clave en el establecimiento de criterios de clonalidad en esta patología 23 .

El cariotipo complejo se pudo documentar en nuestra serie en el 8,6\% (7 casos), comparado con reportes en general alrededor del 10\% en la mayoría de las series de SMD24. Es fundamental entender que el cariotipo complejo es el resultado de un proceso de múltiples etapas con acumulación secuencial de anormalidades, llamada evolución del cariotipo, desentrañando la acumulación gradual de anormalidades secundarias con el punto de partida de una anomalía primaria ${ }^{24,25}$. En algunos casos, la evolución del cariotipo puede observarse si se efectúan análisis citogenéticos seriados, como pudo apreciarse en nuestro estudio en varios casos de seguimiento con evolución clonal; así mismo, se observaron criterios de respuesta citogenética en 8 
de nuestros 22 casos tratados con hipometilantes, como lo estipulado en los criterios de respuesta citogenética del IWG ${ }^{26}$. La correlación de mortalidad de acuerdo con el grupo de riesgo también guardó correlación, siendo mayor en los grupos de riesgo alto y muy alto.

Las alteraciones citogenéticas simples más comunes en SMD las constituyen: del( $5 q)$, pérdida de $Y$, monosomía 7 o del(7q), trisomía 8 y del(20q)27,28; en nuestra serie se apreció con mayor frecuencia: del(5q) y -Y en el $6,1 \%$ (5 casos). La pérdida del cromosoma $Y$ se asocia a un buen pronóstico, es común en los hombres, en particular en los mayores, sin trastornos hematológicos, y no se cree que desempeñe un papel en la patogénesis del SMD en revisiones actuales ${ }^{29}$. Se encontró del(20q), del(11q), del(17q) y +8 en el 3,7\%. Deleciones del brazo largo del cromosoma 20 se presentaron de manera acorde a lo reportado, son esperadas en menos del 5\% de casos; cuando se encuentran como única anomalía, se asocia con un pronóstico favorable ${ }^{30}$. La trisomía del 8 se presenta en cifras menores al $10 \%$ de casos SMD y tiene su importancia en el Sistema Internacional de Puntuación Pronóstica, donde se considera un riesgo intermedio. La trisomía del cromosoma 8 se ha asociado con una mayor expresión de los genes antiapoptóticos en comparación con las células normales y puede dar como resultado una ventaja selectiva sobre los precursores hematopoyéticos normales ${ }^{3,31}$. No obstante, en correlación de grupos de riesgo citogenético y mortalidad, se apreció un 45\% de los fallecidos con clasificación favorable y un $35 \%$ en grupo de alto riesgo citogenético, esto quizás en relación con el corto seguimiento del grupo en torno a los 30 meses.

Sin embargo, existe una variedad mucho mayor de anomalías citogenéticas raras en MDS. En un estudio que investigó una cohorte de más de 2 mil pacientes con SMD, se identificaron un total de 684 categorías citogenéticas diferentes. El amplio espectro de categorías citogenéticas menos frecuentes incluye ganancias de los cromosomas 1 o 1q, 14 o 14q, ganancias o pérdidas del cromosoma 11, 13, 21, o pérdida de un cromosoma $\mathrm{X}$, asociando un pronóstico más adverso a estas tres últimas ${ }^{32}$. En nuestra revisión pudimos encontrar en el rango del $1 \%$ alteraciones no típicas del SMD, dadas por $-22,-13-11$, del $(10 q), 14 p+, 15 q+$ en el $1,2 \%$. No tenemos conocimientos sobre el impacto pronóstico de las variables citogenéticas raras encontradas en nuestra serie. Se debe entender que la falta de validez estadística causada por el bajo número de casos, en la mayoría de las series, ha establecido que las anomalías citogenéticas raras no se consideran específicamente por el IPSS-R y se han asignado al subgrupo intermedio del IPSS-R. Estos y otros ejemplos de anomalías citogenéticas raras hacen hincapié en la necesidad de ampliar y optimizar los modelos pronósticos existentes.

\section{Conclusiones}

Nuestro estudio presenta la serie más grande de pacientes con síndrome mielodisplásico documentada hasta ahora en Colombia, con 81 casos en manejo integral de diagnóstico, tratamiento y seguimiento, evidenciando en aspectos clínicos de clasificación una mayor proporción en casos con SMD de displasia múltiple y SMD de displasia unilinaje, y los SMD no clasificados, con menor proporción de SMD EB 1 y 2. En la estratificación pronóstica predominaron los casos de riesgo bajo e intermedio. La presencia de cariotipo clonal se apreció en el $43 \%$ de los casos, siendo mayor a los reportes regionales. Las frecuencias de alteraciones numéricas y estructurales propias de SMD estaban en correlación con la literatura. La expresión de anomalías atípicas en nuestro estudio plantea una mayor expansión de la base de datos citogenéticos en la literatura. Recomendamos crear un consenso revisor permanente en relación con patrones citogenéticos de SMD asociados a hallazgo de baja frecuencia.

\section{Referencias}

1. Ma X, Does M, Raza A, Mayne ST. Myelodysplastic syndromes: incidence and survival in the United States. Cancer. 2007;109(8):1536-42

2. Rollison $D E$, Howlader $N$, Smith $M T$, Strom SS, Merritt WD, Ries LA, et al. Epidemiology of myelodysplastic syndromes and chronic myeloproliferative disorders in the United States, 20012004, using data from the NAACCR and SEER programs. Blood. 2008;112(1):45-52.

3. Cogle $C R$, Craig BM, Rollison DE, List AF. Incidence of the myelodysplastic syndromes using a novel claims-based algorithm: high number of uncaptured cases by cancer registries. Blood. 2011;117(26):7121-5

4. McQuilten ZK, Wood EM, Polizzotto MN, Campbell LJ, Wall M, Curtis DJ, et al. Underestimation of myelodysplastic syndrome incidence by cancer registries: results from a population-based data linkage study. Cancer. 2014;120(11):1686-94.

5. Swerlow SH, Campo E, Harris NL, Jaffe ES, Pileri SA, Stein H, et al. WHO classification of tumours of haematopoietic and lymphoid tissues. Lyon: IARC Press; 2008.

6. Pang WW, Pluvinage JV, Price EA, Sridhar K, Arber DA, Greenberg PL, et al. Hematopoietic stem cell and progenitor cell 
mechanisms in myelodysplastic syndromes. Proc Natl Acad Sci USA. 2013;110(8):3011-6.

7. Will B, Zhou L, Vogler TO, Ben-Neriah S, Schinke C, Tamari R, et al. Stem and progenitor cells in myelodysplastic syndromes show aberrant stage-specific expansion and harbor genetic and epigenetic alterations. Blood. 2012;120(10):2076-86

8. Woll PS, Kjällquist U, Chowdhury O, Doolittle $H$, Wedge DC Thongjuea S, et al. Myelodysplastic syndromes are propagated by rare and distinct human cancer stem cells in vivo. Cancer Cell. 2014;25(6):794-808

9. Genovese G, Kähler AK, Handsaker RE, Lindberg J, Rose SA Bakhoum SF, et al. Clonal hematopoiesis and blood-cancer risk inferred from blood DNA sequence. $N$ Engl J Med 2014;371(26):2477-87.

10. Haase $D$, Germing U, Schanz J, Pfeilstöcker M, Nösslinger T, Hildebrandt B, et al. New insights into the prognostic impact of the karyotype in MDS and correlation with subtypes: evidence from a core dataset of 2124 patients. Blood. 2007;110 (13):4385-95

11. Pozdnyakova O, Miron PM, Tang G, Walter O, Raza A, Woda $B$, et al. Cytogenetic abnormalities in a series of 1,029 patients with primary myelodysplastic syndromes: a report from the US with a focus on some undefined single chromosomal abnormalities. Cancer. 2008;113(12):3331-40.

12. Mora E, Espinoza D, Casas C, Abello V, Solano M. Caracterización clínica de los pacientes con síndrome mielodisplásico. Acta Med Colomb. 2016;41:36-41

13. Greenberg PL, Tuechler H, Schanz J, Sanz G, GarciaManero G, Solé $F$, et al. Revised international prognostic scoring system for myelodysplastic syndromes. Blood 2012;120(12):2454-65.

14. Bejar R, Levine R, Ebert BL. Unraveling the molecular pathophysiology of myelodysplastic syndromes. J Clin Oncol. 2011;29(5):504-15.

15. American College of Medical Genetics. Standards and guidelines for clinical genetics laboratories. 2009.

16. Association for Clinical Cytogenetics. Professional guidelines for clinical cytogenetics. Hematooncology best practice guidelines. 2007

17. Shaffer LG, McGowan-Jordan J, Schmid M, editors. ISCN 2013. An International System for Human Cytogenetic Nomenclature (2013). Karger; 2013.

18. Alberbide JA. Experiencia argentina en síndromes mielodisplásicos. Hematología. 2016;20(Número Extraordinario)

19. Ma X, Does M, Raza A, Mayne ST. Myelodysplastic syndromes: incidence and survival in the United States. Cancer. 2007;109(8):1536-42.

20. Urlich $G$. The epidemiology of the mielodysplasics syndromes Clinical Leukemias. 2008;2(1):34-8.
21. Belli CB, Pinheiro RF, Bestach $Y$, Larripa IB, da Silva Tanizawa RS, Alfonso G, et al. Myelodysplastic syndromes in South America: a multinational study of 1080 patients. Am J Hematol. 2015;90(10):851-8.

22. Davidoff AJ, Weiss SR, Baer MR, Ke X, Hendrick F, Zeidan A, et al. Patterns of erythropoiesis-stimulating agent use among Medicare beneficiaries with myelodisplasic syndromes and consistency with clinical guidelines. Leuk Res. 2013;37(6):675-80.

23. Schuck A, Götte M, Neukirchen J, et al. Treatment with azacitidine: a retrospective study evaluating the real life clinical course and impact on infectious complications. Blood. 2015;126:1684-90

24. Haase D, Germing U, Schanz J, et al. New insights into the prognostic impact of the karyotype in MDS and correlation with subtypes: evidence from a core dataset of 2124 patients. Blood. 2017;110:4385-95

25. Bernasconi P. Molecular pathways in myelodysplastic syndromes and acute myeloid leukemia: relationships and distinctionsa review. Br J Haematol. 2008;142(5):695-708.

26. Solé F, Luño E, Sanzo C, Espinet B, Sanz GF, Cervera J, et al. Identification of novel cytogenetic markers with prognostic significance in a series of 968 patients with primary myelodysplastic syndromes. Haematologica. 2005;90(9):1168-78.

27. Cheson BD, Greenberg PL, Bennett JM, Lowenberg B, Wijermans PW, Nimer SD, et al. Clinical application and proposal for modification of the International Working Group (IWG) response criteria in myelodysplasia. Blood. 2006;108(2):419-25.

28. Bacher $U$, Haferlach $T$, Kern $W$, Weiss $T$, Schnittger $S$, Haferlach $C$. The impact of cytomorphology, cytogenetics, molecular genetics, and immunophenotyping in a comprehensive diagnostic workup of myelodysplastic syndromes. Cancer. 2009;115(19):4524-32.

29. Lewis S, Oscier D, Boultwood J, Ross F, Fitchett M, Rack K, et al. Hematological features of patients with myelodysplastic syndromes associated with a chromosome $5 q$ deletion. Am J Hematol. 1995;49(3):194-200.

30. Wiktor A, Rybicki BA, Piao ZS, Shurafa M, Barthel B, Maeda K, et al. Clinical significance of $Y$ chromosome loss in hematologic disease. Genes Chromosomes Cancer. 2000;27(1):11-6.

31. Bench AJ, Nacheva EP, Hood TL, Holden JL, French L, Swanton $S$, et al. Chromosome 20 deletions in myeloid malignancies: reduction of the common deleted region, generation of a PAC/BAC contig and identification of candidate genes. UK Cancer Cytogenetics Group (UKCCG). Oncogene. 2000;19(34):3902-13.

32. Arber DA, Orazi A, Hasserjian R, Thiele J, Borowitz MJ, Le Beau MM, et al. The 2016 revision to the World Health Organization classification of myeloid neoplasms and acute leukemia. Blood. 2016:127(20):2391-405

33. Bacher U, Schanz J, Braulke F, Haase D. Rare cytogenetic abnormalities in myelodysplastic syndromes. Mediterr J Hematol Infect Dis. 2015;7(1):e2015034 